\title{
The Problem of Countability of Highest Ordinals
}

\author{
Alexey Pavlovich Kulaichev \\ Department of System Analysis, Moscow State University, Moscow, Russia
}

Email address:

akula@mail.ru

\section{To cite this article:}

Alexey Pavlovich Kulaichev. The Problem of Countability of Highest Ordinals. Pure and Applied Mathematics Journal. Vol. 5, No. 4, 2016, pp. 108-112. doi: 10.11648/j.pamj.20160504.14

Received: March 16, 2016; Accepted: March 25, 2016; Published: June 28, 2016

Abstract: In this study we use the alternate point of view on the structure of ordinals, according to which each ordinal is the union of non-intersecting foregoing segments of ordinals of equal exponentiation. Each ordinal $\omega^{n}=\omega_{1} \times \omega_{2} \times \ldots \times \omega_{n}$ is seen as the union $\omega^{n}=\bigcup_{i=1}^{\omega^{n-j}} \omega_{i}^{j}$ for any $j=1, n-1$ instead traditional union of foregoing intersecting segments of ordinals of consistently increasing exponentiation $\omega^{n}=\bigcup_{i=1}^{n-1} \omega^{i}$. The first form corresponds to the geometric representation of ordinal $\omega^{n}$ as an infinite $n$-dimensional matrix. According traditional formulation $\omega^{\omega}=\bigcup_{i=1}^{\omega} \omega^{i}$, thus $\omega^{\omega}$ is $\omega$-countable union of countable ordinals so $\omega^{\omega}$ is countable. According to alternate formulation $\omega^{\omega}=\bigcup_{i=1}^{\omega^{\omega}} \omega_{i}^{n}$ for any $n$, thus $\omega^{\omega}$ is $\omega^{\omega}$-union of ordinals and the findings will be different. These findings are: 1) the proof of countability of countable union of countable ordinals can not be directly or inductively transferred to its first limit $\omega^{\omega}$-union; 2) $\omega^{\omega}$ seems to be the first uncountable ordinal with its power is equal to continuum; 3) the subsequent ascending degrees of $\omega$-exponentiation of $\omega^{\omega}$, i.e. $\omega^{\omega^{\omega}}$, $\omega^{\omega^{\omega^{\omega}}}, \ldots$, correspond to consecutive $\boldsymbol{\aleph}_{2}, \boldsymbol{\aleph}_{3}, \boldsymbol{\aleph}_{4}, \ldots$ cardinals; 4) from here it also follows the direct justification of continuum hypothesis. Our study shows that in the domain of transfinite sets different points of view and its findings have the legal right to coexist as Nels Bohr's principle of complementarity in physics.

Keywords: Set Theory, Ordinals, Mathematical Induction, Absolute Truth

\section{Introduction}

(1). Ordinals or ordinal type sets except usual attributes, such as cardinality and well-orderliness, also possess certain structure as they consist of consecutive segments. And there can be different points of view on structure of ordinals. For example according traditional interpretation ordinal $\omega^{\omega}$ is defined as countable sum or union of intersecting countable ordinals, therefore $\omega^{\omega}$ is also countable, we quote [4]: « $\omega^{\omega}$ is countable so far as $\omega^{\omega}=\lim \omega^{v}=\lim \left\{\omega^{1}, \omega^{2}, \omega^{3}, \ldots\right\}$ it is the number, which owing to equalities $1+\omega=\omega$, $1+\omega+\omega^{2}=\omega(1+\omega)=\omega^{2}$, etc. can be written down [by analytical transformations] as: $\omega^{\omega}=1+\omega+\omega^{2}+\omega^{3}+\ldots=$ $\sum_{v=1}^{\omega} \omega^{v} »$ [end of quote]. However, there is absolutely overlooked that the summation of ordinals in its ascending order from left to right is identically equal to the last on the right greatest ordinal due to the non-commutativity of addition operation for ordinals. So the above expression for $\omega^{\omega}$ is not the proof but only the tautology $\omega^{\omega}=\omega^{\omega}$.

Other proof of $\omega^{\omega}$ countability is also based on analytical transformation with decomposition of ordinals on other basis, we quote [7]: $« \alpha=\omega^{2}+\omega 5+9$ is the decomposition of $\alpha$ on $\omega$ basis. To spread out the same number on basis 2 , it is enough to notice that $\omega=\lim 2^{n}=2^{\omega}$. Then $\omega^{2}=\left(2^{\omega}\right)^{2}=$ $2^{\omega 2}, \omega 5=2^{\omega+2}+2^{\omega}$, from where we get $\alpha=2^{\omega 2}+2^{\omega+2}+2^{\omega}$ 
$+2^{3}+2^{0}$. By the same way we get $\omega^{\omega}=2^{\omega^{2}} \gg$ [ end of quote]. Taking into account $\omega^{2}=2^{\omega 2}=4^{\omega}=\omega$ we can continue $\omega^{\omega}$ $=2^{\omega^{2}}=2^{\omega}=\omega$.

The last view of $\omega^{\omega}$ structure is significantly different from the previous one and the both of them contradict the standard description of ordinals in Cantor normal form [2]. Besides it is doubtful that such formal transformations are applicable to any ordinals because many other properties of operations with finite numbers aren't transferred to ordinals, e.g.: $1+\omega \neq \omega+1,2 \omega \neq \omega 2$ and so on. Note also that such formal, inductive "proofs" can be generated the next, for example: since $2^{\omega}=4^{\omega}=\ldots=n^{\omega}=\omega$ and $\omega^{\omega}=\lim n^{\omega}$ then $\omega^{\omega}=\omega$.

(2). Moreover, it is well known that in some sequence of ordinals $\{\alpha, \ldots, \beta, \ldots, \chi\}$ some property $P$, which is inherent to all ordinals $\beta<\chi$, is not transferred to $\chi$ if $\chi$ is the limit ordinal for all $\beta<\chi$. The examples of such "intolerable" properties in $\{1,2,3, \ldots, \omega\}$ for its $\omega$-limit are: 1) $Q(n)$ is " $n$ is the number represented by finite digits" but $\neg Q(\omega) ; 2)$ $R(n)$ is " $(n+1) / n>1$ " but $\neg R(\omega)$; 3) $S(n)$ is $" n+1=1+n$ " but $\neg S(\omega) ; 3)$ and so on. Below we also give the examples of not transferred properties for ordinals of higher power.

In such a situation the proof of any property $P(\chi)$ has to be made by noninductive means. The same also concerns to the property "ordinal $\chi$ is countable".

(3). Let us look at the structure of ordinals with an alternative point of view, where each ordinal is the union of non-intersecting sets. Ordinal $\omega$ is $\omega=\bigcup_{n=1}^{\omega} n$, $\omega^{2}=\bigcup_{n=1}^{\omega} \omega_{n}, \omega^{3}=\bigcup_{n=1}^{\omega} \omega_{n}^{2}$ or $\omega^{3}=\bigcup_{n=1}^{\omega^{2}} \omega_{n}$ and so on. And finally, $\omega^{\omega}=\bigcup_{n=1}^{\omega^{\omega}} \omega_{n}^{i}$ for any $i=1,2,3, \ldots$. We see that any $\omega^{i}, i=1,2,3, \ldots$ is at its maximum the $\omega$-union of $\omega^{i-1}$. But $\omega^{\omega}$ can not be at its maximum the union of $\omega^{\omega-1}$ because $\omega^{\omega-i}=\omega^{\omega}$ and for any $i=1,2,3, \ldots$. Let us notice that this point of view ascends to Cantor considering of $\omega^{2}$ ordinal structure [1].

Thus, in a case of $\omega^{\omega}$ we note the leap from all $\omega$ countable-unions to $\omega^{\omega}$-union. Hence the proof of countability of $\omega$-countable-union of countable ordinals can not be directly or inductively transferred to its first $\omega^{\omega}$ union.

\section{Two Methods of Renumbering of Elements of Ordinals}

The fist infinite ordinal $\omega$ designates the set of natural numbers which is countable by definition.

Ordinal $\omega 2$ is the limit for the sequence $1,2,3,4, \ldots, \omega$, $\omega+1, \omega+2, \omega+3, \ldots$ and the proof of its countability is made by direct reordering of its elements therefore it is reduced to the countable sequence $1, \omega, 2, \omega+1,3, \omega+2,4$, $\omega+3, \ldots$. The same method is applicable for subsequent ordinals $\omega 3, \omega 4, \omega 5, \ldots$.

For ordinal $\omega^{2}$ being a limit for the sequence $\omega, \omega 2$, $\omega 3, \ldots$ the above mentioned method of direct reordering is inapplicable and the proof of its countability is made by Cantor pairing function [1], which is equivalent to counterdiagonal renumbering of two-dimensional and infinite in two directions matrix which includes the elements of its non-intersecting subsets $\{1,2,3, \ldots\},\{\omega, \omega+1, \omega+2, \ldots\}$. $\{\omega 2, \omega 2+1, \omega 2+2, \ldots\}, \ldots$.

For ordinal $\omega^{3}$ the corresponding set can be represented by a 3-dimensional and infinite in three directions matrix. And since this ordinal it may be possible to use two various methods to proof its countability.

Method 1 is based on counterdiagonal renumbering or Cantor tuple function [8]. For $\omega^{3}$ we have the counterdiagonal planes in a 3-dimensional matrix. For any subsequent $\omega^{n}$ we have the $n$-1-dimensional counterdiagonal hyperplanes in the corresponding $n$-dimensional matrixes. The number of elements $m_{n, k}$ in such counterdiagonal hyperplanes increase according to the law of figurate numbers [3]:

$$
m_{n, k}=(k+1)(k(n-2)+2) / 2,
$$

where $k=0,1,2, \ldots$ is the order number of counterdiagonal hyperplane.

Method 2 or the method of step-by-step reducing of dimensionality. As ordinal $\omega^{3}$ includes $\omega$ ordinals $\omega^{2}$ then on the first step we perform counterdiagonal renumbering of $\omega$ two-dimensional $\omega^{2}$ matrixes, therefore we obtain the single $\omega^{2}$ matrix which on the second step is reduced by counterdiagonal renumbering to single natural set. To proof the countability of any $\omega^{n}$ it demands to perform $n-1$ of similar steps to decrease dimensions of initial matrix.

\section{Two Opposite Conclusions Concerning $\omega^{\omega}$ Countability}

Using these two methods it is possible to come to the opposite conclusions concerning $\omega^{\omega}$ countability.

Proposition-1. $\omega^{\omega}$ is not countable.

The proof is made directly by method 1 .

(1). Because $\omega^{\omega}$ is representable by infinite matrix with infinite dimensions, therefore its first counterdiagonal hyperplane contains infinite number of elements $m_{\omega, 1}=\omega$, so here the process of renumbering will be finished.

(2). From the other side, the Cantor pairing function [1] which uniquely encode two natural numbers $k_{1}, k_{2}$ into a single natural number $\pi\left(k_{1}, k_{2}\right)$ is defined by

$$
\pi\left(k_{1}, k_{2}\right)=0.5\left(k_{1}+k_{2}\right)\left(k_{1}+k_{2}+1\right)+k_{2}
$$

This definition is recursively generalized to the Cantor tuple function [8]

$$
\pi^{(n)}\left(k_{1}, k_{2}, k_{3}, \ldots, k_{n}\right)=\pi\left(\pi^{(n-1)}\left(k_{1}, k_{2}, k_{3}, \ldots, k_{\mathrm{n}-1}\right), k_{\mathrm{n}}\right)
$$

For $n=\omega$, taking into account that $\omega-1=\omega$ we have 
$\pi^{(\omega)}\left(k_{1}, k_{2}, k_{3}, \ldots, k_{\omega}\right)=\pi\left(\pi^{(\omega-1)}\left(k_{1}, k_{2}, k_{3}, \ldots, k_{\omega-1}\right), k_{\omega}\right)$

$$
=\pi\left(\pi^{(\omega)}\left(k_{1}, k_{2}, k_{3}, \ldots, k_{\omega}\right), k_{\omega}\right)
$$

So we get the equation which is unsolvable and the renumbering of elements of $\omega^{\omega}$ set is not possible.

Thereby the proposition-1 have been proved from both sides.

We can formulate objection and counter-objection against these reasoning.

\section{Objection.}

Let us look at formula (1). According to method 1 for $n=$ $\omega$, i.e. for any $k$-counterdiagonal-hyperplane in $\omega^{\omega}$, we have:

$$
m_{\omega, k}=(k+1)(k \omega+2) / 2=k^{2} \omega+k \omega+k+1,
$$

e.g.: $m_{\omega, 2}=5 \omega+3, m_{\omega, 3}=10 \omega+4, m_{\omega, 4}=17 \omega+5, \ldots$, $m_{\omega, \omega}=\omega^{3}+\omega^{2}+\omega+1$.

Since in $\omega^{\omega}$ there are only $\omega$ counterdiagonal hyperplanes then $\omega^{\omega}$ is the countable union of countable sets.

\section{Counter-objections.}

(1). According to formula (5) $\omega^{\omega}$ is the union of $\omega$ sets raised not more then to 3 -th power. This goes against the nature of $\omega^{\omega}$ set and against the Cantor normal form [2].

(2). Except counterdiagonal renumbering of elements of $\omega^{\omega}$ set it is possible the alternate renumbering «on covers», e.g. for 2-dimentional infinite $\omega^{2}$ matrix (Fig. 1).

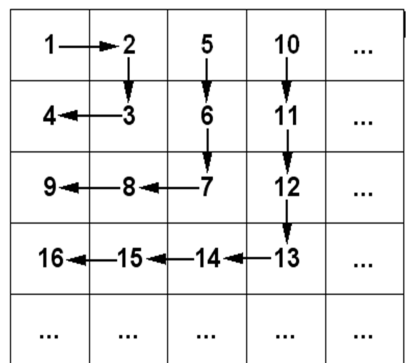

Fig. 1. Alternate order of renumbering of 2-dimentional infinite $\omega^{2}$ matrix.

Each $k=0,1,2,3, \ldots$ cover in $n$-dimensional $\omega^{n}$ matrix includes $e_{n, k}=(k+1)^{n}-k^{n}$ elements, i.e. in $\omega^{\omega}$ matrix each $k$-cover includes $e_{\omega, k}=(k+1)^{\omega}-k^{\omega}=\omega$ - $\omega=0$ elements.

Summary. Thus we see that depending on renumbering order of $\omega^{\omega}$ elements we get different conclusions concerning $\omega^{\omega}$. But absolute mathematical truth should not depend on renumbering order of $\omega^{\omega}$ matrix elements. It once again shows that any formula or property derived for natural numbers, as well as formula (5), can not be directly or inductively transferred to its $\omega$ limit, as it have been stated in the introduction. Therefore similar transfer in the abovestated objection is incorrect, as well as the objection itself. On the contrary, the proof (2) of the proposition-1 clearly shows that the rule of renumbering of previous $\omega^{n}$ ordinals, which is going back to Cantor, can not be extended to their $n=\omega$-limit.

Proposition-2. $\omega^{\omega}$ is countable.

The proof is made by method 2 of step-by-step decrease of dimensions in infinite sequence $\omega^{2}, \omega^{3}, \omega^{4}, \ldots$, ordinals enclosed each other up to $\omega^{\omega}$.

We can formulate four objections against these reasoning. Objections.

(1). This proof implicitly uses inductive procedure to transfer the property of all elements of ordered set $\omega^{2}, \omega^{3}$, $\omega^{4}, \ldots$ to its unattainable $\omega^{\omega}$ limit. That can lead to a wrong result as it noted in the introduction.

(2). To prove that $\omega^{3}$ is countable it is previously necessary to renumber $\omega$ copies of $\omega^{2}$ matrixes in $\omega^{3}$ having received as a result a single $\omega^{2}$ matrix which then is reduced to one natural row, that is to execute $\omega+1$ of counterdiagonal renumbering of $\omega^{2}$ matrixes. For $\omega^{4}$ it is necessary in the same way to renumber $\omega$ copies of $\omega^{3}$ matrixes totally execute $\omega^{2}+\omega+1$ renumbering of $\omega^{2}$ matrixes. For $\omega^{n}$ it is necessary to execute $\omega^{n-2}+\omega^{n-3}+\ldots+\omega+1$ of such renumbering of $\omega^{n-2}$ matrixes. For $\omega^{\omega}$ it is necessary to execute not less than $\omega^{\omega-2}$ renumbering of $\omega^{2}$ matrixes, that is equal to $\omega^{\omega}$ renumbering. Thereby to prove that $\omega^{\omega}$ is countable it is necessary to execute such number of renumbering which countability we are going to prove. Therefore this proof, which implicitly uses the still an unproven subject of the proof, is unconvincing.

(3). Let's look at the problem from the other side. $\omega^{\omega}$ set contains not less than $\omega^{\omega-2}$ of $\omega^{2}$ sets. Thereby if we reduce each $\omega^{2}$ set to $\omega$ set we obtain $\omega^{\omega-2}$ set i.e. again initial $\omega^{\omega}$ set as unsolvable "begging the question" arisen.

(4). The method 1 is based on Cantor tuple function which allows us unambiguously to calculate for each element of $\omega^{n}$ its position in a resultant natural sequence. On the contrary the inverse Cantor tuple function allows us uniquely to decompose natural numbers set to $\omega^{n}$ set. Method 2 for any ordinal $\omega^{n}$ does exactly the same, i.e. it formulates another tuple function with the same properties. But proof 2 of proposition-1 states that for ordinal $\omega^{\omega}$ the tuple function and its inversion does not exist.

On the adduced four arguments we did not find any counter-objections.

Summary. We considered two antithesis about countability of $\omega^{\omega}$ ordinal and their proof. On ratios of possible objections and counter-objections the proposition-1 is more convincing. For a final choice from these two alternatives it is desirable to find such property of $\omega^{\omega}$ ordinal, proceeding from the used paradigm of its matrix representation, which proof can't be disproved neither by method 1 , nor by method 2 . 


\section{The Cardinality of $\omega^{\omega}$}

Theorem-1. $\omega^{\omega}$ cardinality is equal to cardinality of powerset of natural numbers or continuum $2^{\aleph_{0}}=c$

Proof

Powerset of natural numbers includes: all subsets of one number, all subsets of two numbers and so on up to all subsets of infinite natural numbers.

On the other hand, all subsets of two numbers are representable in a two-dimensional infinite $\omega^{2}$ matrix (Fig. 2) and the first line of this matrix contains subsets of one element.

\begin{tabular}{|c|c|c|c|c|c|}
\hline$\{1\}$ & $\{2\}$ & $\{3\}$ & $\{4\}$ & $\{5\}$ & $\cdots$ \\
\hline$\{1,2\}$ & $\{1,3\}$ & $\{1,4\}$ & $\{1,5\}$ & $\{1,6\}$ & $\cdots$ \\
\hline$\{2,3\}$ & $\{2,4\}$ & $\{2,5\}$ & $\{2,6\}$ & $\{2,7\}$ & $\cdots$ \\
\hline$\{3,4\}$ & $\{3,5\}$ & $\{3,6\}$ & $\{3,7\}$ & $\{3,8\}$ & $\cdots$ \\
\hline$\{4,5\}$ & $\{4,6\}$ & $\{4,7\}$ & $\{4,8\}$ & $\{4,9\}$ & $\cdots$ \\
\hline$\cdots$ & $\cdots$ & $\cdots$ & $\cdots$ & $\cdots$ & $\cdots$ \\
\hline
\end{tabular}

Fig. 2. Two-dimensional infinite $\omega^{2}$ matrix represented all subsets of two numbers.

All subsets of three numbers are representable in 3dimensional infinite $\omega^{3}$ matrix at which the first plane contains subsets of one and two elements.

And so on to all subsets of natural numbers which is representable by infinite matrix with infinite number of dimensions and this matrix is corresponded to $\omega^{\omega}$ set.

Thereby this theorem have been proved.

Consequence 1. Continuum hypothesis follows from this proof because in the ascending and continuous sequence of all countable transfinite ordinals $\omega, \ldots, \omega^{2}, \ldots, \omega^{3}, \ldots$, $\omega^{n}, \ldots$ ordinal $\omega^{\omega}$ is the first noncountable one and it is equal to cardinality of powerset of natural numbers $2^{\aleph_{0}}=c$. Therefore its cardinality is the first one after $\boldsymbol{\aleph}_{0}$ i.e. $\boldsymbol{\aleph}_{1}$.

Consequence 2. The subsequent ordinals of ascending $\omega$ exponentiation of $\omega^{\omega}$ represent powerset of ordinals of previous degree of $\omega$-exponentiation of $\omega^{\omega}$ thereby $\omega^{\omega^{\omega}}$, $\omega^{\omega^{\omega^{\omega}}}, \ldots$ correspond to cardinals $\boldsymbol{\aleph}_{2}, \boldsymbol{\aleph}_{3}, \boldsymbol{\aleph}_{4}, \ldots$. Thus, in a case of cardinal $\boldsymbol{\aleph}_{2}=2^{\aleph_{1}}$ we should apply the theorem-1 proof to transfinite matrix of $\omega^{\omega}$-length instead of $\omega$-length and with $\omega^{\omega}$ number of dimensions instead $\omega$ number of dimensions. This matrix represents ordinal $\omega^{\omega^{\omega}}$. And so on.

\section{Discussion}

At the turn of XX century in the history of mathematics it has been occurred the significant but not noticed event. Prior to that mathematics revealed or took from some unknown storage the absolute truths such as multiplication table, squaring the circle, Fourier transform, etc. But in the last third of XIX century in mathematics there were began to appear theories and hypotheses [5]. But any theory represents only some set of initial intuitively or substantially reasonable provisions intended by means of certain rules and conclusions logically convincingly and consistently to explain some properties and phenomena of external or mental world. Thus for the similar explanation may be used several different theories and the adoption of one or another theory is not a consequence of its absolute truth, but a matter of public agreement. One or other social community might prefer one or another theory and follows it in accordance with the freedom of its choice. The same situation has occurred in respect of numerous set theories [9].

Moreover the degree of public recognition of any theory in natural sciences area is determined by predictive adequacy of concrete theory, since these predictions are usually available for further experimental testing. Mathematics is not a science with possibility of experimental verification of its theoretical provisions in area of set theories. Instead of experimental validation of mathematical findings the only criterion of its truth remains the consistency of its conclusions in the framework of the theory itself, but does not the presence of contradictions with the conclusions of other theories.

Further, any result in any theory can not be wrong iff it is not consistent with other results in other theory obtained from a different point of view and using a different proof. Results can be wrong iff the point of view is wrong or the proof includes errors. Indeed it is impossible to disprove the theorems in Euclidean geometry on the base of results in spherical geometry. In such a situation, at the degree of public acceptance of one or another set theory and its results there begin to influence professional consents, political considerations and teleological grounds, which is not always compatible with the dispassionate scientific approach to the evidences [6].

Numerous subtleties of such public consents in most accepted version of set theory force some professors and lecturers clearly to warn their audience about these subtleties in their textbooks [10], we quote: "In fact, we have already reached a dangerous border where visual representation of sets lead to a contradiction", and also: "Here again we come to the dangerous border of paradoxes and so we have to be expressed evasively".

\section{Conclusion}

As we above have seen the question of countability or uncountability of some ordinals also depends on a particular viewpoint on the structure of such ordinals. So in most popular set theory according to the axiom of exponentiation of ordinals it is considered that ordinal $\omega^{n}$ is the union or sum of intersecting segments of ordinals of previous 
sequence of $\omega$-exponentiation, i.e. $\omega^{n}=\bigcup_{i=1}^{n} \omega^{i}$ or $\omega^{n}=\sum_{i=1}^{n} \omega^{i}$ [4] that ascends to Cantor natural form [2]. According to that $\omega^{\omega}=\bigcup_{i=1}^{\omega} \omega^{i}$ and $\omega^{\omega}$ seems like $\omega$ countable union of countable intersecting ordinals, so $\omega^{\omega}$ is also countable. But it is possible the alternate point of view: $\omega^{n}=\bigcup_{i=1}^{\omega^{n-j}} \omega_{i}^{j}$ for any $j=1, n-1$, thus $\omega^{\omega}=\bigcup_{i=1}^{\omega^{\omega}} \omega_{i}^{n}$ for any $n$, so $\omega^{\omega}$ is $\omega^{\omega}$-union of foregoing non-intersecting segments of ordinals of equal exponentiation, that leads to the conclusion that $\omega^{\omega}$ is uncountable.

Our study shows that in the domain of transfinite sets the different points of view and its findings have the legal right to coexist as Nels Bohr's principle of complementarity in physics. Acceptance or rejection of a particular viewpoint in this area is not the subject of absolute truth but the subject of a particular public agreements.

\section{References}

[1] Georg Cantor, 1878, Ein beitrag zur mannigfaltigkeitslehre. Journal fr die reine und angewandte Mathematik, 84, 242258.

[2] Georg Cantor, 1883, Grundlagen einer allge. meinen Mannigfaltigkeitslehre: Ein mathematisch-philo-sophischer Versuch in der Lehre des Unendlichen. Leipzig: Teubner.

[3] Deza, E., Deza, M., 2011, Figurate Numbers. World Scientific.

[4] Felix Hausdorff, 1914, Grundzuge der Mengenlehre. Veit and Company, Leipzig.

[5] Stephen Cole Kleene, 1967, Mathematical logic. N. Y.

[6] Morris Kline, 1980, Mathematics. The loss of certainty. N. Y.

[7] Kuratowski K., Mostowski A., 1967, Set theory. Amsterdam.

[8] Lisi M., 2007, Some remarks on the Cantor pairing function. Le Matematiche, 62 (1), 55-65.

[9] Maydim A. Malkov, 2014, Stairs of Natural Set Theories, Pure and Applied Mathematics Journal. 3 (3), 49-65.

[10] Vereshchagin N. K., Shen A., 2002, Lectures on mathematical logic and theory of algorithms. Moscow, MCNME. 